Acta Cardiologica

\title{
Summary of 2019 ESC Guidelines on chronic coronary syndromes, acute pulmonary embolism, supraventricular tachycardia and dislipidaemias
}

\section{Marc J. Claeys, Yves Vandekerckhove, Bernard Cosyns, Philippe Van de Borne} \& Patrizio Lancellotti

To cite this article: Marc J. Claeys, Yves Vandekerckhove, Bernard Cosyns, Philippe Van de Borne \& Patrizio Lancellotti (2020): Summary of 2019 ESC Guidelines on chronic coronary syndromes, acute pulmonary embolism, supraventricular tachycardia and dislipidaemias, Acta Cardiologica, DOI: $10.1080 / 00015385.2019 .1699282$

To link to this article: https://doi.org/10.1080/00015385.2019.1699282

\section{曲 Published online: 10 Jan 2020.}

Submit your article to this journal 줄

Llll Article views: 33

Q View related articles $\sqsubset$

View Crossmark data $₫$ 


\title{
Summary of 2019 ESC Guidelines on chronic coronary syndromes, acute pulmonary embolism, supraventricular tachycardia and dislipidaemias
}

\author{
Marc J. Claeys ${ }^{\mathrm{a}}$ (D), Yves Vandekerckhove ${ }^{\mathrm{b}}$, Bernard Cosyns ${ }^{c}$, Philippe Van de Borne ${ }^{\mathrm{d}}$ and \\ Patrizio Lancellotti ${ }^{\mathrm{e}}$

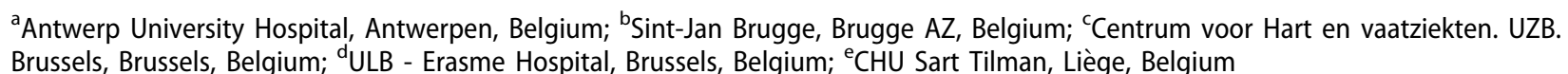

ABSTRACT

During the ESC congress in September 2019 in Paris, the new ESC guidelines were presented and are now available on the ESC website. The new guidelines describe management recommendations on following cardiovascular diseases: chronic coronary syndromes, acute pulmonary embolism, supraventricular tachycardia and dislipidaemias. The present document gives a summary of these guidelines and highlights the most important recommendations and changes in the management of these diseases. It will help to increase awareness about the new guidelines and may stimulate to consult the full document for specific items. Ultimately, the authors hope that this document will enhance implementation of new ESC guidelines in daily clinical practice.
ARTICLE HISTORY

Received 24 November 2019; Accepted 25 November 2019

\section{KEYWORDS}

ESC guidelines; chronic coronary syndromes; acute pulmonary embolism; supraventricular tachycardia; dislipidaemias

\section{ESC guidelines for the diagnosis and management of chronic coronary syndromes (CCS)}

The Guidelines have been revised to focus on CCS instead of stable CAD in order to emphasise the fact that the clinical presentations of CAD can be categorised as either acute coronary syndromes (ACS) or CCS [1]. Six clinical scenarios most frequently encountered in patients have been identified: from naive and asymptomatic patients to symptomatic patients (angor/dyspnea) presenting after an ACS. The pre-test probability (PTP) of CAD based on age, gender and nature of symptoms have undergone major revisions and the concept 'Clinical likelihood of $C A D$ ' has been introduced that utilises also various risk factors of CAD as PTP modifiers (Figures 1 and 2). New recommendations can be divided in three categories:

1. Basic testing, diagnostics, and risk assessment

- Non-invasive functional imaging for myocardial ischaemia or coronary computed tomography (CTA) is recommended (Class I) as the initial test for diagnosing $C A D$ in symptomatic patients in whom obstructive CAD cannot be excluded by clinical assessment alone.
- Exercise ECG is now only recommended for the assessment of exercise tolerance, symptoms, arrhythmias, blood presure (BP) response, and event risk in selected patients. It has been downgraded in II B category as an alternative test to rule-in or rule-out CAD when other non-invasive or invasive imaging methods are not available and in patients on treatment to evaluate control of symptoms and ischaemia.

- It is also recommended that selection of the initial non-invasive diagnostic test be based on the clinical likelihood of CAD and other patient characteristics that influence test performance, local expertise, and the availability of tests.

- Functional imaging for myocardial ischaemia is recommended if coronary CTA has shown CAD of uncertain functional significance or is not diagnostic.

- Invasive angiography is recommended as an alternative test to diagnose CAD in patients with a high clinical likelihood and severe symptoms refractory to medical therapy, or typical angina at a low level of exercise and clinical evaluation that indicates high event risk. Invasive functional assessment must be available and used to evaluate stenoses before

CONTACT Marc J. Claeys marc.claeys@uantwerpen.be Department of Cardiology, University of Antwerp Hospital (Edegem), Wilrijkstraat 102650 Edegem, Belgium

(C) 2020 Belgian Society of Cardiology 


\begin{tabular}{|l|c|c|c|c|c|c|}
\hline & \multicolumn{2}{|c|}{ Typical } & \multicolumn{2}{c|}{ Atypical } & \multicolumn{2}{|c|}{ Non-anginal } \\
\hline Age & Men & Women & Men & Women & Men & Women \\
\hline $30-39$ & $3 \%$ & $5 \%$ & $4 \%$ & $3 \%$ & $1 \%$ & $1 \%$ \\
\hline $40-49$ & $22 \%$ & $10 \%$ & $10 \%$ & $6 \%$ & $3 \%$ & $2 \%$ \\
\hline $50-59$ & $32 \%$ & $13 \%$ & $17 \%$ & $6 \%$ & $11 \%$ & $3 \%$ \\
\hline $60-69$ & $44 \%$ & $16 \%$ & $26 \%$ & $11 \%$ & $22 \%$ & $6 \%$ \\
\hline $70+$ & $52 \%$ & $27 \%$ & $34 \%$ & $19 \%$ & $24 \%$ & $10 \%$ \\
\hline
\end{tabular}

\begin{tabular}{|c|c|}
\hline \multicolumn{2}{|c|}{ Dyspnoea $^{\mathbf{a}}$} \\
\hline Men & Women \\
\hline $0 \%$ & $3 \%$ \\
\hline $12 \%$ & $3 \%$ \\
\hline $20 \%$ & $9 \%$ \\
\hline $27 \%$ & $14 \%$ \\
\hline $32 \%$ & $12 \%$ \\
\hline
\end{tabular}

Figure 1. Pre-test probabilities of obstructive CAD in 15815 symptomatic patients according to age, sex, and the nature of symptoms in a pooled analysis of contemporary data. CAD: coronary artery disease; PTP: pre-test probability. ${ }^{\mathrm{a}}$ In addition to the classic Diamond and Forrester classes, ${ }^{59}$ patients with dyspnoea only or dyspnoea as the primary symptom are included. The regions shaded dark green denote the groups in which non-invasive testing is most beneficial (PTP $>15 \%$ ). The regions shaded light green denote the groups with PTPs of CAD between 5-15\%, in which testing for diagnosis may be considered after assessing the overall clinical likelihood based on the modifiers of PTPs presented in Figure 4.

PTP based on sex, age and nature of symptoms (Table 5)

Decreases likelihood

- Normal exercise ECG

- No coronary calcium by CT

$(\text { Agatston score }=0)^{2}$

Increases likelihood

- Risk factors for CVD

(dyslipidaemia, diabetes, hypertension, smoking, family history of CVD)

- Resting ECG changes (Q-wave or ST-segment/ T-wave changes)

- LV dysfunction suggestive of CAD

- Abnormal exercise ECG ${ }^{a}$

- Coronary calcium by $\mathrm{CT}^{\mathrm{a}}$

\section{Clinical likelihood of CAD}

Figure 2. Determinants of the clinical likelihood of obstructive CAD.

revascularization, unless very high grade (>90\% diameter stenosis).

- Invasive coronary angiography with the availability of invasive functional evaluation should be considered (Class IIA) for confirmation of the diagnosis of CAD in patients with an uncertain diagnosis on non-invasive testing.

- Coronary CTA should be considered as an alternative to invasive angiography if another noninvasive test is equivocal or non-diagnostic.

- Importantly, CTA is not recommended (Class III) when extensive coronary calcification, irregular heart rate, significant obesity, inability to cooperate with breath-hold commands, or any other conditions make good image quality unlikely.

2. Antithrombotic therapy in patients with CCS
- Addition of a second antithrombotic drug to aspirin for long-term secondary prevention should be considered (Class IIA) in patients with a high risk of ischaemic events and without high bleeding risk for patients with CCS and in sinus rhythm and may be considered (Class IIB) in patients with at least a moderately increased risk of ischaemic events and without high bleeding risk.

- In patients with CCS and atrial fibrillation (AF) a direct oral anticoagulant (DOAC) is recommended (Class I) in preference to a vitamin $\mathrm{K}$ antagonists (VKA) in eligible patients.

- In patients with CCS and post-PCl, who are eligible for a DOAC, it is recommended that a DOAC (apixaban $5 \mathrm{mg}$ b.i.d., dabigatran $150 \mathrm{mg}$ b.i.d., edoxaban $60 \mathrm{mg}$ o.d., or rivaroxaban $20 \mathrm{mg}$ o.d.) is used in preference to a VKA in combination with antiplatelet therapy. (Class I)

- In the same population, several Class IIA specific recommendations are provided for specific antithrombotic treatment: when rivaroxaban is used and concerns about high bleeding risk prevail over concerns about stent thrombosis or ischaemic stroke, rivaroxaban $15 \mathrm{mg}$ o.d. should be considered in preference to rivaroxaban $20 \mathrm{mg}$ o.d. for the duration of concomitant single or dual antiplatelet therapy. When dabigatran is used and concerns about high bleeding risk prevail over concerns about stent thrombosis or ischaemic stroke, dabigatran $110 \mathrm{mg}$ b.i.d. should be considered in preference to dabigatran $150 \mathrm{mg}$ b.i.d. for the duration of concomitant single or dual antiplatelet therapy.

- After uncomplicated $\mathrm{PCl}$, early cessation ( $\leq 1$ week) of aspirin, and continuation of dual therapy with OAC and clopidogrel, should be 


\section{Suspected PE in a patient without haemodynamic instabilitya}

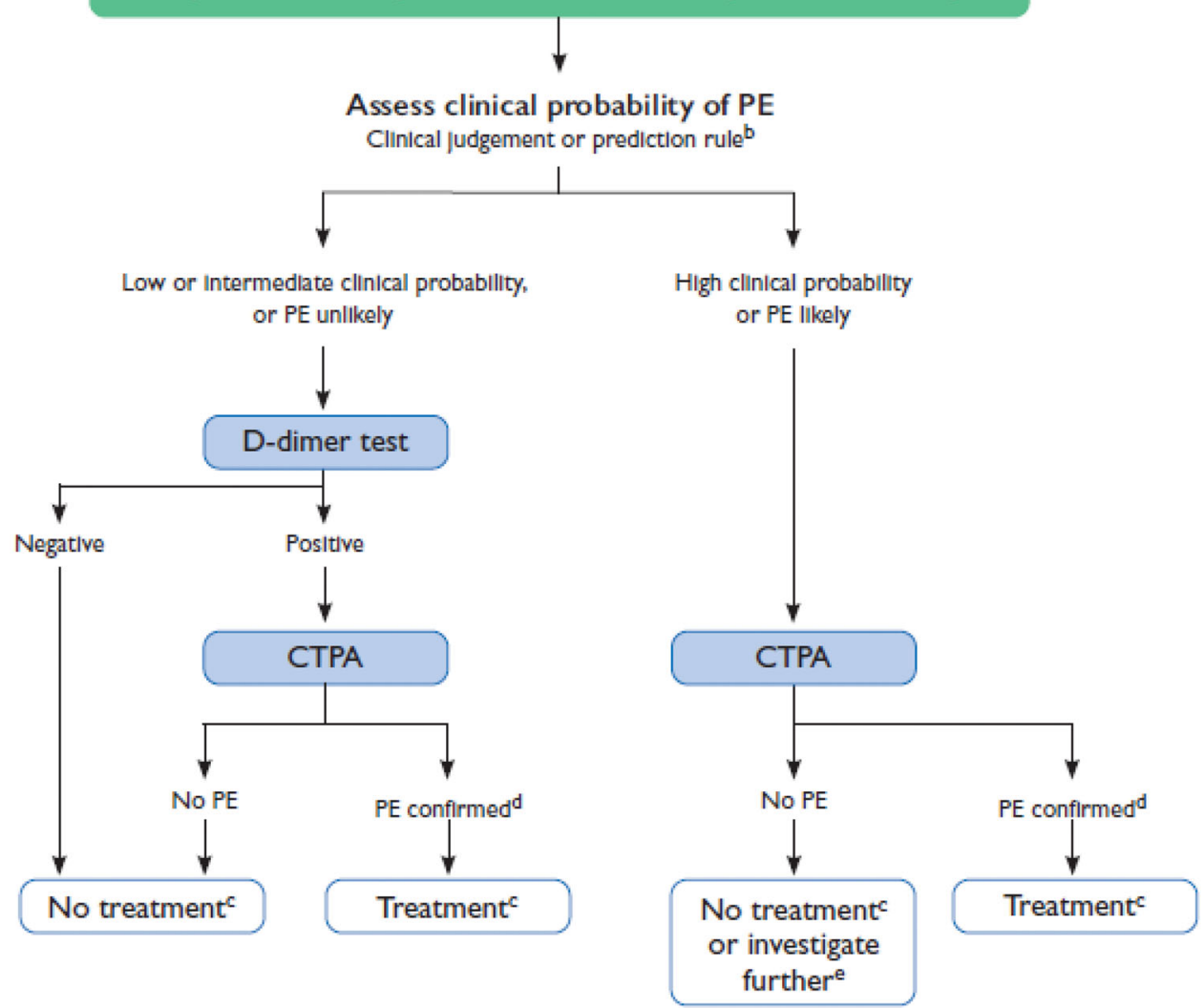

Figure 3. Diagnostic algorithm for patients with suspected pulmonary embolism. CTPA: computed tomography pulmonary angiography; PE: pulmonary embolism.

considered if the risk of stent thrombosis is low or if concerns about bleeding risk prevail over concerns about risk of stent thrombosis, irrespective of the type of stent used.

- Triple therapy with aspirin, clopidogrel, and an OAC for $\geq 1$ month should be considered when the risk of stent thrombosis outweighs the bleeding risk, with the total duration ( $\leq 6$ months) decided upon according to the assessment of these risks and clearly specified at hospital discharge.

3. Other pharmacological therapy

- Concomitant use of a proton pump inhibitor is recommended (Class I) in patients receiving aspirin monotherapy, DAPT, or OAC monotherapy who are at high risk of gastrointestinal bleeding.

- Lipid-lowering drugs: for patients at very high risk who do not achieve their goals on a maximum tolerated dose of statin and ezetimibe, combination with a PCSK9 inhibitor is Class I.

- The sodium-glucose co-transporter 2 inhibitors empagliflozin, canagliflozin, or dapagliflozin are recommended (Class I) in patients with diabetes mellitus and cardiovascular disease (CVD) as well as glucagon-like peptide-1 receptor agonist (liraglutide or semaglutide).

- ACE inhibitors should be considered (Class IIA) in CCS patients at very high risk of cardiovascular adverse events.

\section{ESC guidelines for the management of acute pulmonary embolism (PE)}

Pulmonary embolism (PE) remains a major life-threatening condition. In the new ESC guidelines 2019 on the management of $P E$, the focus has been on the optimal diagnosis, evaluation and treatment with the goal to decrease variation in practice in treating $P E$ and improve patient outcomes [2].

The ESC Task Force also selected a set of key rules to guide physicians, the most important and those that were new or revisited are listed below.

1. Diagnosis

- In patients presenting with haemodynamic instability, perform bedside transthoracic 
echocardiography as a fast, immediate step to differentiate suspected high-risk PE from other acute life-threatening situations.

- Use recommended, validated diagnostic algorithms for $\mathrm{PE}$, including standardised assessment of (pre-test) clinical probability and D-dimer testing (Figure 3).

- A D-dimer test, using an age-adjusted cut-off or adapted to clinical probability, should be considered as an alternative to the fixed cutoff level (lla).

- The finding of a proximal DVT in patients suspected of having PE on compression venous ultrasonography (CUS) is considered sufficient to confirm PE further risk assessment risk assessment should be considered to guide management (Ila).

2. Risk assessment

- Stratify patients with suspected or confirmed $P E$, based on the presence of haemodynamic instability, to identify those at high risk of early mortality (I).

- Confirmation of PE in a patient, without haemodynamic instability, must be followed by further risk assessment involving clinical findings, evaluation of the size and/or function of the right ventricle, and laboratory biomarkers as appropriate (Figure 3).

- Assessment of the RV by imaging or laboratory biomarkers should be considered, even in the presence of a low pulmonary embolism severity index. PESI or a sPESI of 0 (Ila).

3. Treatment in the acute phase (Figure 4)

- If you suspect acute PE, institute anticoagulation therapy as soon as possible, while the diagnostic workup is ongoing, unless the patient is bleeding or has absolute contraindications to this therapy

- Administer systemic thrombolytic therapy to patients with high-risk PE (cardiac arrest, obstructive shock, persistent hypotension) (I).

- Rescue thrombolytic therapy is recommended for patients who deteriorate haemodynamically (I).

- Surgical embolectomy or catheter-directed treatment should be considered as alternatives to rescue thrombolytic therapy for patients who deteriorate haemodynamically (lla).

- If anticoagulation is initiated parenterally in a patient without haemodynamic instability, prefer LMWH or fondaparinux over UFH (I).

- When oral anticoagulation is initiated in a patient with PE who is eligible for a DOAC (apixaban, dabigatran, edoxaban, or rivaroxaban), a DOAC is the recommended form of anticoagulant treatment (I).

- Set-up of multidisciplinary teams for management of high-risk and selected cases of intermediate-risk PE should be considered, depending on the resources and expertise available in each hospital (Ila).

4. Chronic treatment and prevention of recurrence

- Administer therapeutic anticoagulation for $\geq 3$ months to all patients with PE (I).

- Routine clinical evaluation is recommended 3-6 months after acute PE (I).

- Discontinue therapeutic oral anticoagulation after 3 months in patients with first PE secondary to a major transient/reversible risk factor (I).

- Indefinite treatment with a VKA is recommended for patients with antiphospholipid antibody syndrome (I).

- Extended anticoagulation should be considered for patients with no identifiable risk factor for the index PE event (Ila).

- Extended anticoagulation should be considered for patients with a persistent risk factor other than antiphospholipid antibody syndrome (lla).

- Extended anticoagulation should be considered for patients with a minor transient/reversible risk factor for the index PE event (Ila).

- A reduced dose of apixaban or rivaroxaban should be considered after the first 6 months (lla).

- It is recommended that symptomatic patients with mismatched perfusion defects on a V/Q scan $>3$ months after acute PE are referred to a pulmonary hypertension/CTEPH expert centre, taking into account the results of echocardiography, natriuretic peptide, and/or cardiopulmonary exercise testing (I).

5. $\mathrm{PE}$ in cancer

- In patients with PE and cancer edoxaban or rivaroxaban should be considered as an alternative to $L M W H$, with the exception of patients with gastrointestinal cancer (Ila). In addition, extended anticoagulation (beyond the first 6 months) should be considered for an indefinite period or until cancer is cured.

\section{ESC guidelines for the management of supraventricular tachycardia (SVT)}

This is the first update of the guidelines on management of supraventricular tachycardia since 2003 [3]. The most important messages are: 


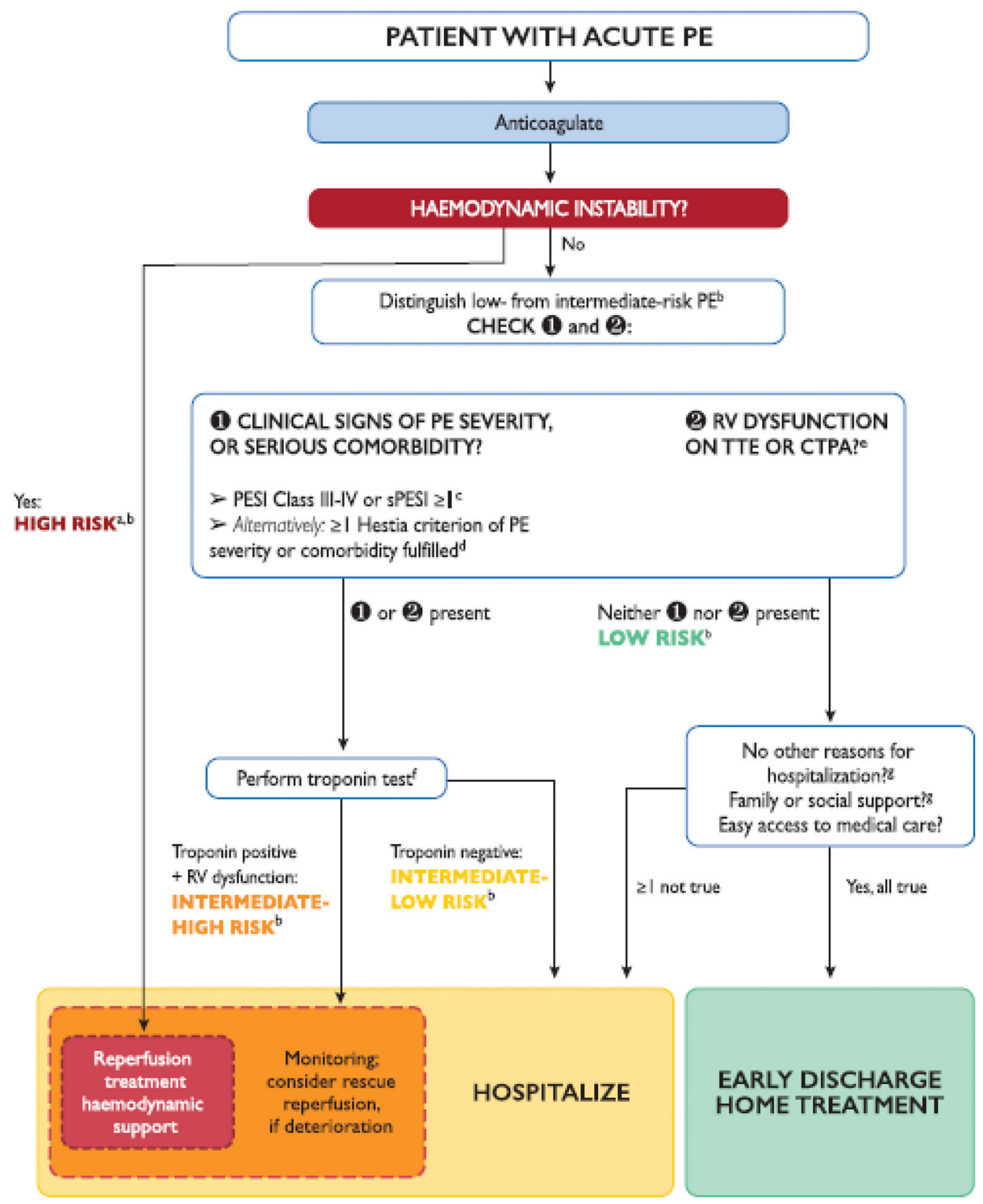

Figure 4. Management algorithm for patients with acute pulmonary embolism. CTPA: computed tomography pulmonary angiography; PE: pulmonary embolism, PESI: Pulmonary Embolism Severity Index; RV: right ventricular; sPESI: Simplified Pulmonary Embolism Severity Index; TTE: trans thoracic echocardiography.

1. The treatments of choice for acute therapy of supraventricular tachycardia are vagal manoeuvers and adenosine IV (IB) and may also provide important diagnostic information; verapamil, diltiazem and beta-blockers receive recommendation Ila. The registration of a twelve lead ECG during tachycardia and also during acute treatment are recommended (IC).

2. Sinus tachycardia

- Inappropriate sinus tachycardia:

- first evaluation and treatment of reversible underlying causes (IC).
- beta blockers and/or Ivabradine should be considered (Ila).

- catheter ablation and calcium antagonists are not mentioned.

- Sinus atrial re-entry tachycardia:

When not responsive to drugs, catheter ablation should be considered (Ila).

3. In all re-entrant supraventricular tachycardia and most focal supraventricular tachycardia catheter ablation is offered as initial treatment to patients after having explained in detail potential risks and benefits. 


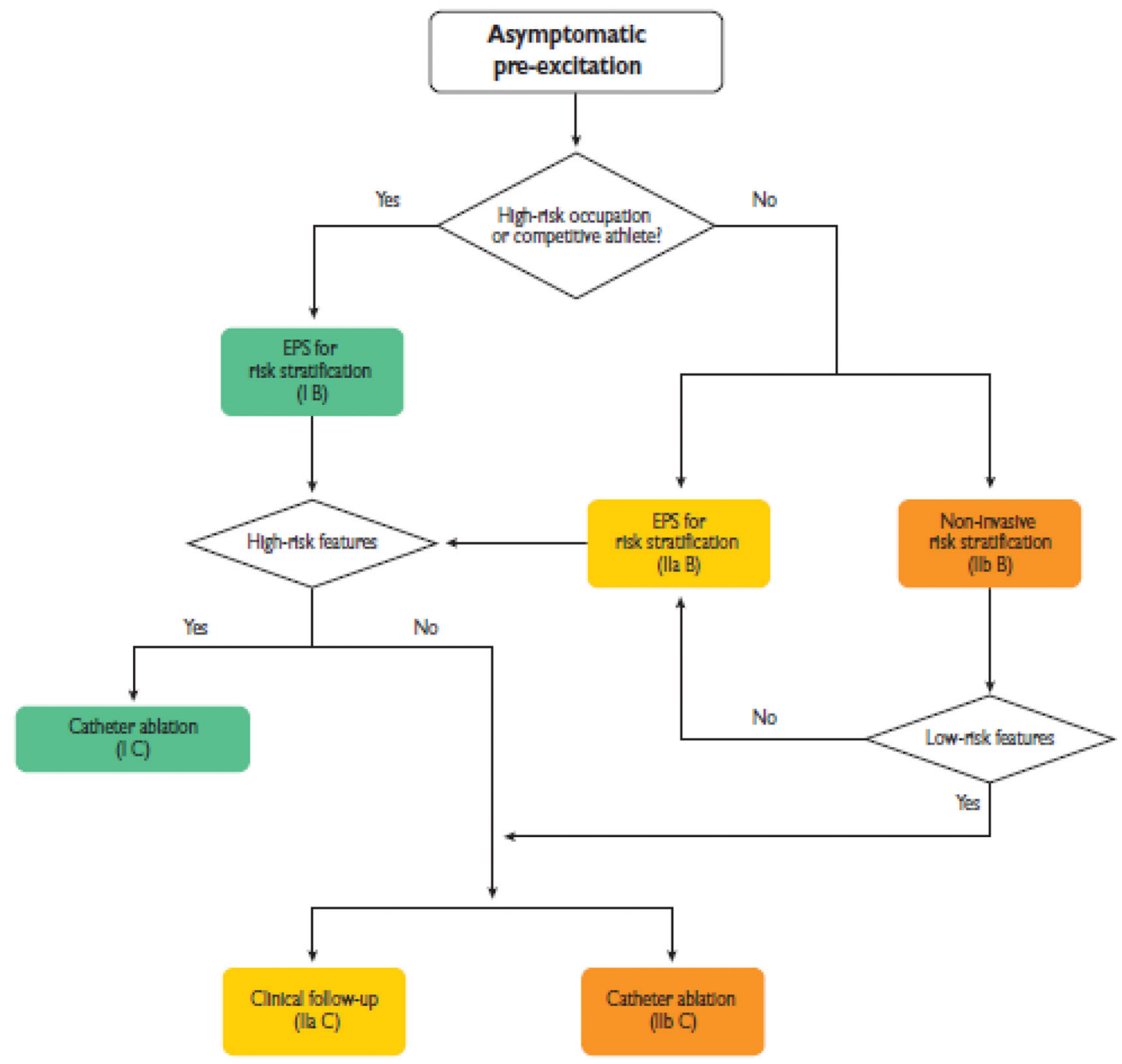

Figure 5. Risk stratification and therapy of patients with asymptomatic pre-excitation.

4. Ablation of post AF ablation atrial tachycardia should be deferred $\geq 3$ months after ablation when possible.

5. Catheter ablation in symptomatic and recurrent AVNRT (recommendation IB) can now be realised with almost no risk of AV block.

6. In macro re-entrant atrial tachycardia (flutter) without atrial fibrillation anticoagulation should be considered (Ila). But the threshold for initiation has not been established.

7. Asymptomatic pre-excitation (WPW) see risk stratification diagram.

- One in five patients develops arrhythmias during follow-up. Risk of cardiac arrest is 2.4 per 1000 person-years.

- Non-invasive tests may be considered (Ilb) risk stratification and its predictive ability remains modest.

- Invasive testing (EPS) is recommended in patients with asymptomatic pre-excitation and high-risk occupation or competitive athletes (IB) and should be considered in all others (Ila).

- Catheter ablation is recommended in asymptomatic patients with shortest RR interval $\leq 250$ ms during AF, refractory periods of accessory pathway $<250 \mathrm{~ms}$, multiple accessory pathways and if inducible AVRT(IC) (Figure 5).

- Catheter ablation should be considered in asymptomatic low-risk patients (Illb).

8. Supraventricular tachycardia in pregnant woman:

- During the first trimester it is recommended that all antiarrhythmic drugs are avoided (IC).

- In SVT without WPW beta- I selective blocker (except atenolol) or Verapamil should be considered (Ila).

- In WPW without structural heart disease or ischaemic heart disease flecaïnide or propafenone should be considered (lla).

- Fluoroless ablation should be considered in cases of drug-refractory or poorly tolerated 
B Treatment goal

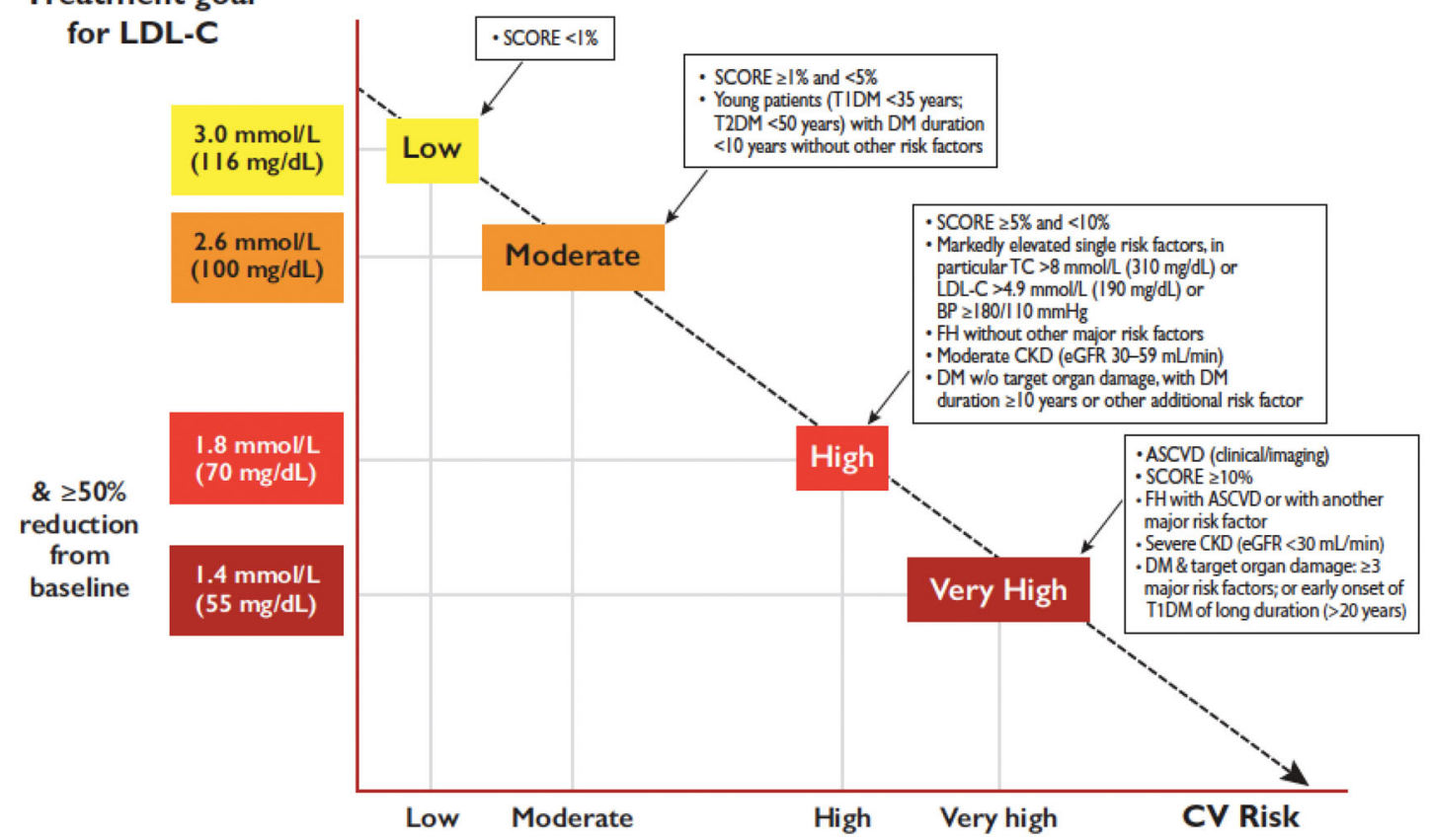

Figure 6. Cardiovascular risk categories and treatment goals. CT: computed tomography; CKD: chronic kidney disease; DM: diabetes mellitus; eGFR: estimated glomerular filtration rate; FH: familial hypercholesterolaemia; SCORE: systematic coronary risk estimation; TIA: transient ischaemic accident. Target organ damage is defined as microalbuminuria, retinopathy or neuropathy.

supraventricular tachycardia, in experienced centres (Ila).

9. Tachycardia-induced cardiomyopathy (TCM)

- TCM is one of the very few reversible causes of heart failure and should be considered in all new cases of LV dysfunction (IB).

- Ablation is the treatment of choice in tachy cardiomyopathy due to supraventricular tachycardia (IB).

- AV nodal ablation with subsequent biventricular or HIS bundle pacing (ablate + pace) should be considered if the supraventricular tachycardia cannot be cured (IC).

\section{ESC guidelines for the management of dyslipidaemias}

Several recent landmark studies made the European Society of Cardiology (ESC) and the European Atherosclerosis Society (EAS) dyslipidemia revise their management guidelines [4]. This is a short summary of the main modifications with this new document, in comparison to the previous Joint European Society of Cardiology (ESC)/European Atherosclerosis Society (EAS) Dyslipidaemia guidelines published 3 years ago.

- Cardiovascular risk levels

The different established risk levels (Figure 6) are based on the best available evidence. It should be stressed that very high-risk levels not only apply to patients with established atherosclerotic cardiovascular disease (ASCVD) but also to some high-risk patients in the setting of primary prevention.

In subjects at low to moderate risk, arterial plaque burden by means of ultrasonography (carotid and/or femoral) or coronary artery calcium score are recommended risk modifiers.

- Lower low-density lipoprotein (LDL) goals to be achieved in high and very high-risk patients

It is now recommended that very high-risk patients, in secondary but also in primary prevention, achieve LDL-C levels of $<55 \mathrm{mg} / \mathrm{dL}(<1.4 \mathrm{mmol} / \mathrm{L})$ and at least $50 \%$ reduction from baseline LDL-C levels. In high-risk patients, the LDL-C goal is $<70 \mathrm{mg} / \mathrm{dL}(<1.8 \mathrm{mmol} / \mathrm{L})$ and at least $50 \%$ reduction from baseline LDL-C levels. A LDL-C goal of $<1.0 \mathrm{mmol} / \mathrm{L}(<40 \mathrm{mg} / \mathrm{dL})$ may be considered if a patient experience a second vascular event within 2 years (not necessarily in the same vascular territory) on maximally tolerated statin therapy (Figure 6).

\section{- Combination therapy}

To achieve such low targets, the new ESC/EAS guidelines emphasise the importance of combination treatment, first with ezetimibe and then with a PCSK9 inhibitor. In patients with an acute coronary syndrome, adding a PCSK9 inhibitor early after the event (if 
possible, during hospitalization) should be considered. In these patients, if the LDL-C goal is not achieved after 4-6 weeks despite maximally tolerated statin therapy and ezetimibe, a PCSK9 inhibitor should also be added.

- n-3 polyunsaturated fatty acids ( $n-3$ PUFAs - fish oils) in triglyceride management

Statin treatment remains the first choice for managing high triglycerides (TG, $>200 \mathrm{mg} / \mathrm{dL}$ or $2.3 \mathrm{mmol} / \mathrm{L}$ ). However when despite statin treatment high-risk patients continue to present persistently elevated TG (between $135-499 \mathrm{mg} / \mathrm{dL}$ or 1.5 and $5.6 \mathrm{mmol} / \mathrm{L}$ ), $\mathrm{n}-3$ PUFAs (icosapent ethyl $2 \times 2 \mathrm{~g}$ daily) are recommended. Fenofibrate or bezafibrate may be considered in high-risk patients with an LDL-C at goal as a result of statins, but in whom TG remain $>200 \mathrm{mg} / \mathrm{dL}$ or $>2.3 \mathrm{mmol} / \mathrm{L}$.

- Lipoprotein(a)

$\mathrm{Lp}$ (a) should be measured at least once in all adults. Mendelian randomisation and epidemiologic studies suggest very high $L p(a)$ indicate a lifetime atherosclerotic cardiovascular risk comparable to heterozygous familial hypercholesterolaemia. Nowadays, only PCSK9 inhibitors can reduce $L p(a)$ by $25-30 \%$, but new therapies are awaited.

- Dyslipidaemia after 75 years

Before 75 years, statins are recommended for primary prevention depending on the level of risk. After 75 years, initiation of statin treatment for primary prevention may be considered (Class IIb) when at high risk or above. In case of renal function impairment or risk for drug interaction, the low initiation dose is progressively up titrated to achieve LDL-C goals.

\section{Disclosure statement}

No potential conflict of interest was reported by the authors.

\section{ORCID}

Marc J. Claeys (iD http://orcid.org/0000-0002-6628-9543

\section{References}

[1] Knuuti J, Wijns W, Saraste A, et al. 2019 ESC Guidelines for the diagnosis and management of chronic coronary syndromes. Eur Heart J. 2019. DOI: 10.1093/eurheartj/ehz425

[2] Konstantinides SV, Meyer G, Becattini C, et al. 2019 ESC Guidelines for the diagnosis and management of acute pulmonary embolism developed in collaboration with the European Respiratory Society (ERS). Eur Heart J. 2019. DOI:10.1093/eurheartj/ehz405

[3] Brugada J, Katritsis DG, Arbelo E, et al. 2019 ESC Guidelines for the management of patients with supraventricular tachycardia: the Task Force for the management of patients with supraventricular tachycardia of the European Society of Cardiology (ESC). Eur Heart J. 2019. DOI:10.1093/eurheartj/ehz467

[4] Mach F, Baigent C, Catapono AL, et al. 2019 ESC/EAS Guidelines for the management of dyslipidaemias: lipid modification to reduce cardiovascular risk. Eur Heart J. 2020;41:111-188. 